\title{
One-pot synthesis of magnetic nanoclusters enabling atherosclerosis-targeted magnetic resonance imaging
}

This article was published in the following Dove Press journal:

International Journal of Nanomedicine

2I May 2014

Number of times this article has been viewed

\author{
Aastha Kukreja' \\ Eun-Kyung Lim ${ }^{2-4}$ \\ Byunghoon Kang' \\ Yuna Choi ${ }^{2}$ \\ Taeksu Lee' \\ Jin-Suck Suh ${ }^{2,3}$ \\ Yong-Min Huh ${ }^{2,3}$ \\ Seungjoo Haam ${ }^{1,3}$ \\ 'Department of Chemical and \\ Biomolecular Engineering, College \\ of Engineering, ${ }^{2}$ Department of \\ Radiology, College of Medicine, Yonsei \\ University, Seoul, Republic of Korea; \\ ${ }^{3}$ YUHS-KRIBB Medical Convergence \\ Research Institute, Seoul, Republic \\ of Korea; ${ }^{4} \mathrm{BioNanotechnology}$ \\ Research Center, Korea Research \\ Institute of Bioscience and \\ Biotechnology, Daejeon, Republic of \\ Korea
}

\begin{abstract}
In this study, dextran-encrusted magnetic nanoclusters (DMNCs) were synthesized using a one-pot solution phase method for detection of atherosclerosis by magnetic resonance imaging. Pyrenyl dextran was used as a surfactant because of its electron-stabilizing effect and its amphiphilic nature, rendering the DMNCs stable and water-dispersible. The DMNCs were $65.6 \pm 4.3 \mathrm{~nm}$, had a narrow size distribution, and were superparamagnetic with a high magnetization value of $60.1 \mathrm{emu} / \mathrm{g}$. Further, they showed biocompatibility and high cellular uptake efficiency, as indicated by a strong interaction between dextran and macrophages. In vivo magnetic resonance imaging demonstrated the ability of DMNCs to act as an efficient magnetic resonance imaging contrast agent capable of targeted detection of atherosclerosis. In view of these findings, it is concluded that DMNCs can be used as magnetic resonance imaging contrast agents to detect inflammatory disease.
\end{abstract}

Keywords: magnetic nanocrystal, magnetic resonance imaging, atherosclerosis, macrophages, dextran

\section{Introduction}

Magnetic nanoparticles have excellent potential in the early diagnosis of a number of diseases by magnetic resonance (MR) imaging. ${ }^{1-6}$ Recently, magnetic nanoparticles have been synthesized using various well defined approaches, such as thermal decomposition and coprecipitation processes. Magnetic nanoparticles synthesized by thermal decomposition have well defined crystallinity and magnetic sensitivity. ${ }^{7-15}$ However, they are not soluble in aqueous phase, so have limited biological application. ${ }^{16,17}$ Complicated surface modification processes are often used to make magnetic nanoparticles hydrophilic in order to improve their colloidal stability, thereby increasing their circulation time in the body and targeting a specific organ/tissue or tumor with a high affinity. ${ }^{18-26}$ For example, Fang et al recently utilized triethoxysilylpropylsuccinic anhydride for surface modification of iron oxide nanoparticles to make them water-soluble and biocompatible; they further coupled these with amine-functionalized poly(ethylene glycol), leaving the amine groups free and allowing for further conjugation to various biomolecules for targeting. ${ }^{22}$ Despite stabilization of such nanoparticles, surface modification can significantly increase their size, resulting in limited tissue distribution and metabolic clearance from the body. ${ }^{27,28}$ It is therefore crucial that we devise contemporary synthetic methodologies for uncomplicated synthesis of magnetic nanoclusters with water-dispersibility, monodispersity, and ease of synthesis. ${ }^{29,30}$ In this work, we used a facile one-pot strategy to synthesize hydrophilic dextran-encrusted magnetic nanoclusters (DMNCs) enabling atherosclerosis-targeted MR imaging. 
Specifically, DMNCs can be used for accurate diagnosis of atherosclerosis via MR imaging, and this is rooted in the strong interaction between the dextran present on the surface of DMNCs with the macrophages present in the atherosclerotic lesions. ${ }^{31-37}$ Pyrenyl dextran, used as a surfactant, was first synthesized using dextran and 1-pyrenebutyric acid, as previously reported. ${ }^{32,33}$ The DMNCs were then synthesized with iron precursors and pyrenyl dextran in the solution phase. We confirmed the physicochemical properties of DMNCs and their usefulness as MR imaging agents for detection of atherosclerosis using in vitro/in vivo systems (Figure 1).

\section{Materials and methods Materials}

All chemicals and reagents were of analytical grade and used as received. 1-Pyrenebutyric acid, 1,3-dicyclohexylcarbodiimide (DCC), 4-dimethylaminopyridine, anhydrous dimethyl sulfoxide, triethylamine, iron (III) chloride, sodium acetate, diethylene glycol (DEG), and ethylene glycol were purchased from Sigma-Aldrich (St Louis, MO, USA). Dextran T10 (molecular weight 10,000 Da) was purchased from Pharmacia Biotech (Pfizer, New York City, NY, USA). Phosphate-buffered saline (10 mM, pH 7.4), fetal bovine serum, and Dulbecco's Modified Eagle Medium were obtained from Gibco (Carlsbad, CA, USA), and the dialysis membrane (molecular weight cutoff 3,500) was obtained from Pierce (Thermo Fisher Scientific, Waltham, MA, USA). Ultrapure deionized water was used for all synthesis procedures.

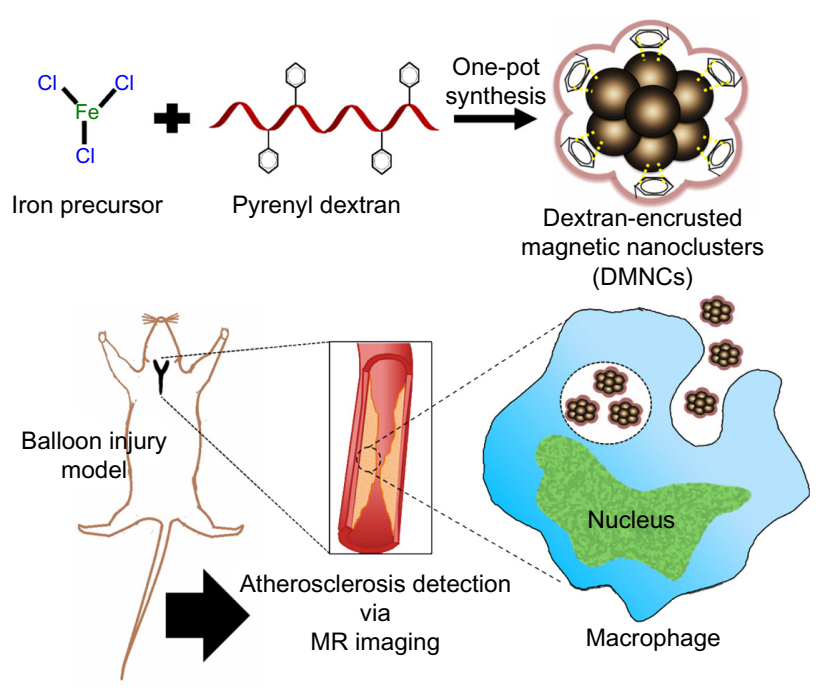

Figure I Schematic representation of the synthesis of DMNCs enabling atherosclerosis-targeted MR imaging.

Abbreviations: DMNCs, dextran-encrusted magnetic nanoclusters; MR, magnetic resonance.

\section{Synthesis of pyrenyl dextran}

Pyrenyl dextran, an amphiphilic copolymer, was formed using the recommended bioconjugation technique. ${ }^{32,33}$ The hydroxyl group of dextran (molecular weight 10,000 Da) was linked to the carboxylic group of 1-pyrenebutyric acid (molecular weight 288.34 Da) using DCC and DMAP as the coupling agent and catalyst, respectively (Figure 1). A mixture of $0.1 \mathrm{mmol}$ of dextran, $4.2 \mathrm{mmol}$ of 1-pyrenebutyric acid, $0.5 \mathrm{mmol}$ of DCC, $0.5 \mathrm{mmol}$ of 4-dimethylaminopyridine, and $0.2 \mathrm{mmol}$ of triethylamine dissolved in $60 \mathrm{~mL}$ of dimethyl sulfoxide was reacted for 48 hours at room temperature followed by lyophilization. To remove the byproducts, the resulting mixture was then filtered through a cellulose acetate filter (pore size $200 \mathrm{~nm}$ ) after being dissolved in excess deionized water. For further purification, dialysis was performed over 7 days. The purified product was lyophilized under vacuum and stored for later use. Characterization of the as-synthesized pyrenyl dextran was completed using Fourier-transform infrared spectroscopy (Excalibur series, Varian Inc., Palo Alto, CA, USA) and ${ }^{1} \mathrm{H}$ nuclear magnetic resonance (NMR) spectrometry $(400 \mathrm{mHz}$, INOVA 400 NMR spectrometer, Varian Inc.).

\section{Synthesis of DMNCs}

First, $1 \mathrm{mmol}$ of iron chloride and $20 \mathrm{mg}$ of as-synthesized pyrenyl dextran were dissolved in a solution containing $15 \mathrm{~mL}$ of DEG and $5 \mathrm{~mL}$ of ethylene glycol. ${ }^{38} \mathrm{After}$ complete solubilization, $1.50 \mathrm{~g}$ of sodium acetate was added and the mixture was continuously stirred. The resulting mixture was then sealed in a Teflon stainless steel autoclave at $220^{\circ} \mathrm{C}$ for 6 hours. After cooling, the viscous black product was washed with ethanol and deionized water three times followed by magnetic separation.

\section{Cell culture}

RAW264.7 cells from a macrophage cell line were obtained from the American Type Culture Collection (Manassas, VA, USA). The cells were maintained in Dulbecco's Modified Eagle Medium supplemented with $10 \%$ fetal bovine serum and $1 \%$ antibiotic-antimycotic at $37^{\circ} \mathrm{C}$ with $5 \% \mathrm{CO}_{2}$.

\section{Cytotoxicity test for DMNCs}

The in vitro cytotoxicity of DMNCs was evaluated by measuring the inhibition of cell growth by a 3-[4,5dimethylthiazol-2-yl]-2,5 diphenyl tetrazolium bromide (MTT) assay of RAW264.7 cells. The cells were seeded in a 96-well plate at a density of $2 \times 10^{4}$ cells per well and allowed to grow overnight. The cells were then treated with 
DMNCs at various concentrations for a further 24 hours. The yellow tetrazolium salt in the MTT solution was reduced to purple formazan crystals in the live cells. Cell viability was determined by calculating the ratio of the intensity of purple formazan in the viable cells treated with DMNCs to the intensity in nontreated control cells. ${ }^{39}$

\section{Cellular uptake of DMNCs}

The uptake of DMNCs was determined by Prussian blue staining. Two million cells per well of the RAW264.7 (macrophage) cell line were incubated overnight with Dulbecco's Modified Eagle Medium in six-well plates. The cells were further incubated with DMNCs $(125 \mu \mathrm{g} / \mathrm{mL})$ in serum-free medium for 8 hours. After washing, the cells were collected in phosphate-buffered saline using a cell scraper. The cells were then stained with Prussian blue stain and viewed under a light microscope. The cells were fixed in 95\% alcohol for 5 minutes followed by immersion in iron staining solution (20\% hydrochloric acid to potassium ferrocyanate, $1: 1)$ for 30 minutes at room temperature. To remove the residual staining solution, the samples were next rinsed with deionized water three times. Finally, the cells were stained with nuclear fast red staining solution for 15 minutes. The cells were then washed three times with deionized water, followed by fixation in increasing concentrations of alcohol and xylene. Tissue samples were analyzed using a virtual microscope (Olympus BX51, Tokyo, Japan) and Olyvia software. A transmission electron microscope was used to confirm internalization of the DMNCs by macrophage cells.

\section{MR imaging procedure}

The MR experiments were performed using a $1.5 \mathrm{~T}$ clinical MR imaging instrument equipped with a micro-47 surface coil (Intera, Philips Medical Systems, Best, the Netherlands). The R2 relaxivities of the DMNCs were measured using the Carr-Purcell-Meiboom-Gill sequence at room temperature: TR, 10 seconds; 32 echoes with 12 msec even echo space; number of acquisitions, 1; a point resolution of $156 \times 156 \mathrm{~mm}$; and section thickness of $0.6 \mathrm{~mm}$. For acquisition of T2-weighted MR images for the DMNC solution, the following parameters were used: resolution $234 \times 234 \mathrm{~mm}$; section thickness $2.0 \mathrm{~mm}$; TE $60 \mathrm{msec}$; TR 4,000 msec; and number of acquisitions, 1 . The relaxivity coefficient $\left(\mathrm{mM}^{-1} \mathrm{~s}^{-1}\right)$ equals the ratio of $\mathrm{R} 2\left(1 / \mathrm{T} 2, \mathrm{~s}^{-1}\right)$ to DMNC concentration. For the in vivo MR imaging experiments, the following parameters were used at room temperature: TR 4,000 msec even echo space; number of acquisitions, 1; point resolution $312 \times 312 \mathrm{~mm}$; section thickness $0.6 \mathrm{~mm}$; and a TE of $60 \mathrm{msec}$. The results are shown as the mean \pm standard deviation.

\section{Animal experiments}

All animal experiments were conducted using SpragueDawley rats and with approval from the International Association for Assessment and Accreditation of Laboratory Animal Care. We used a rat balloon injury model for investigation of atherosclerosis imaging. ${ }^{40,41}$ A 3:1 blend of Zoletil-Rompun was first used to anesthetize the rats (300-350 g). The surgical area below the chin of each rat was then sterilized, and an incision was made using sharp serrated-edge scissors. Next, using fine forceps, a blunt dissection was done to expose all glands. The glands were gently separated to expose the underlying muscular layer; this was followed by separation of the muscular tissue, leading to exposure of the left common carotid artery, the vagus nerve, and other adjacent nerves and vessels, which were then separated. Blunt dissection was further continued to expose the carotid artery bifurcation into the internal and external branches. Next, while the left distal common carotid was tightly tied for hemostasis, one approximately 3-inch 4-0 silk suture was loosely tied around the external carotid artery branch. A Fogarty arterial embolectomy catheter was introduced through the external carotid artery and guided through the common carotid artery down to the aortic arch. The balloon was then slowly inflated with saline to generate slight resistance and the catheter was withdrawn along the full length of the thoracic-abdominal aorta; this procedure was repeated eight times. The catheter was removed after carotid arterial injury, and the external carotid was occluded by closing the skin incision with sutures. The surgical area was swabbed to prevent infection. After 3 weeks, the common carotid arteries were again separated, as previously described, and the common carotid artery was exposed to a $10 \% \mathrm{FeCl}_{3}$ solution for 5 minutes to develop thrombus by means of a chemical burn. The incision was then tightly sutured. We then performed our in vivo MR imaging experiments. DMNCs $\left(4.8 \mathrm{mg}_{\mathrm{Fe}} / \mathrm{kg}\right)$ were administered via intravenous injection into the tail vein. After 5 hours, the common carotid artery was excised and the rats were euthanized. ${ }^{40,41}$

\section{Histological analysis}

A frozen section procedure was performed on the extracted common carotid artery, and the Masson's trichrome and Prussian blue staining methods were used. Using a previously established protocol, we verified the internal and external common carotid artery structures by 
Masson's trichrome staining. ${ }^{42-44}$ In addition, the common carotid artery was stained with Prussian blue dye to confirm the presence of DMNCs at the target sites. The common carotid artery slides were fixed in 95\% alcohol for 5 minutes followed by immersion in iron staining solution (20\% hydrochloric acid to potassium ferrocyanate, 1:1) for 30 minutes at room temperature. To remove the residual staining solution, the samples were then rinsed three times with deionized water. Finally, the tissues were stained with nuclear fast red staining solution for 15 minutes. The tissues were then washed three times with deionized water, and fixed in increasing concentrations of alcohol and xylene. The stained slides were analyzed using a virtual microscope (Olympus BX51) and Olyvia software.

\section{Statistical analysis}

All data represent triplicate experiments, with each experiment yielding comparable results. Statistical evaluation was performed using analysis of variance and the Student's $t$-test. A $P$-value $<0.001$ was considered to be statistically significant.

\section{Results and discussion Synthesis and characterization of pyrenyl dextran}

A modified "one-pot" method was developed for synthesis of water-soluble DMNCs for use as an atherosclerosis targeting MR contrast agent using pyrenyl dextran as a surfactant. Iron (III) chloride was used as a single iron precursor, sodium acetate was used as a reducing agent, and DEG and ethylene glycol were used as the high boiling point polar solvents. Pyrenyl dextran was first synthesized as a stabilizer by esterification of 1-pyrenebutyric acid as a hydrophobic moiety and dextran as a hydrophilic moiety using DCC and 4-dimethylaminopyridine (see Figure S1). ${ }^{32,33}$ Synthesis of pyrenyl dextran was confirmed by Fouriertransform infrared and ${ }^{1} \mathrm{H}-\mathrm{NMR}$ spectra. Generation of its ester group was observed as an absorption peak at $1,727 \mathrm{~cm}^{-1}$ in the Fourier-transform infrared spectra (see Figure S2). ${ }^{1} \mathrm{H}-\mathrm{NMR}$ peaks were observed at $7.71 \mathrm{ppm}, 8.12$ ppm (-CH- from 1-pyrenebutyric acid), $2.03 \mathrm{ppm}\left(-\mathrm{CH}_{2}-\right.$ from 1-pyrenebutyric acid), $4.81 \mathrm{ppm}$ and $4.78 \mathrm{ppm}(-\mathrm{OH}$ from dextran), and $3.40 \mathrm{ppm}$ and $3.71 \mathrm{ppm}\left(-\mathrm{CH}_{2}-\right.$ from dextran, see Figure S3). These results demonstrate the effective synthesis of pyrenyl dextran via the bioconjugation approach. Pyrenyl dextran was selected on the basis of its electrochemical properties, in that it can provide electrons for the iron cations on the magnetic nanocluster surface and can serve as an electrostatic stabilizer. ${ }^{32,33}$

\section{Synthesis of DMNCs}

DMNCs were then fabricated via one-pot synthesis in a solution phase at high temperature for high magnetic sensitivity. This method does not require any extra surface modification steps. In this synthetic process, the above-synthesized pyrenyl dextran is used as a surfactant to provide a stable colloidal dispersion of DMNCs and to avoid aggregation. Ethylene glycol is introduced along with DEG as a polar solvent, because DEG alone leads to formation of a sheetlike structure as compared with the monodispersed magnetic nanoclusters formed when ethylene glycol is used with a DEG to ethylene glycol ratio of $3: 1 .^{38,45,46}$

DMNCs were formed by a previously recommended two-step growth prototype, with nucleation in a supersaturated solution as the first step followed by aggregation into larger secondary particles. ${ }^{47}$ Using this process, addition of sodium acetate appears to be important as it seems to be involved in the reduction of iron (III) chloride to iron oxide. Control experiments were also performed, and showed that $\mathrm{Fe}^{3+}$ could not be reduced in the absence of sodium acetate under the same reaction conditions. In particular, pyrenyl dextran could help in size control as well as increasing the colloidal stability in this synthetic method.

\section{Characterization of DMNCs}

The size and surface charge of the DMNCs as measured by laser scattering was $65.6 \pm 4.3 \mathrm{~nm}$ and $2.3 \pm 0.3 \mathrm{mV}$, respectively. Figure $2 \mathrm{~A}$ and $\mathrm{B}$ shows the transmission electron microscopic and scanning electron microscopic images, respectively. Using a transmission electron microscope, the spherical shape of the DMNCs with a narrow size distribution was observed, along with little aggregation, which

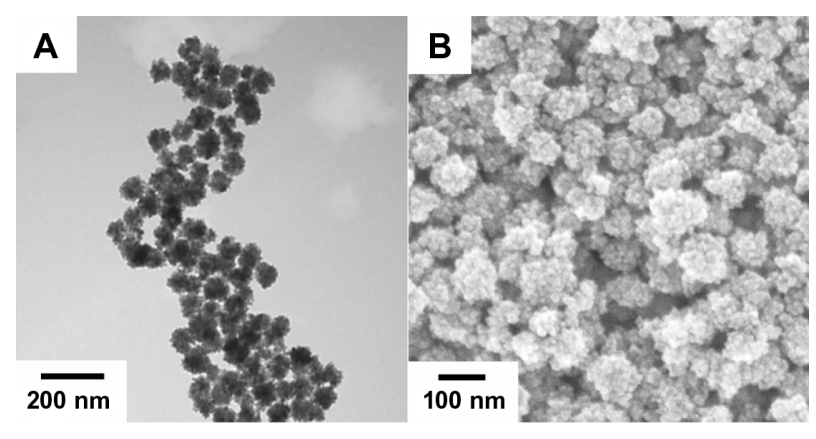

Figure 2 (A) Transmission electron microscopic image and (B) scanning electron microscopic image of dextran-encrusted magnetic nanoclusters. 


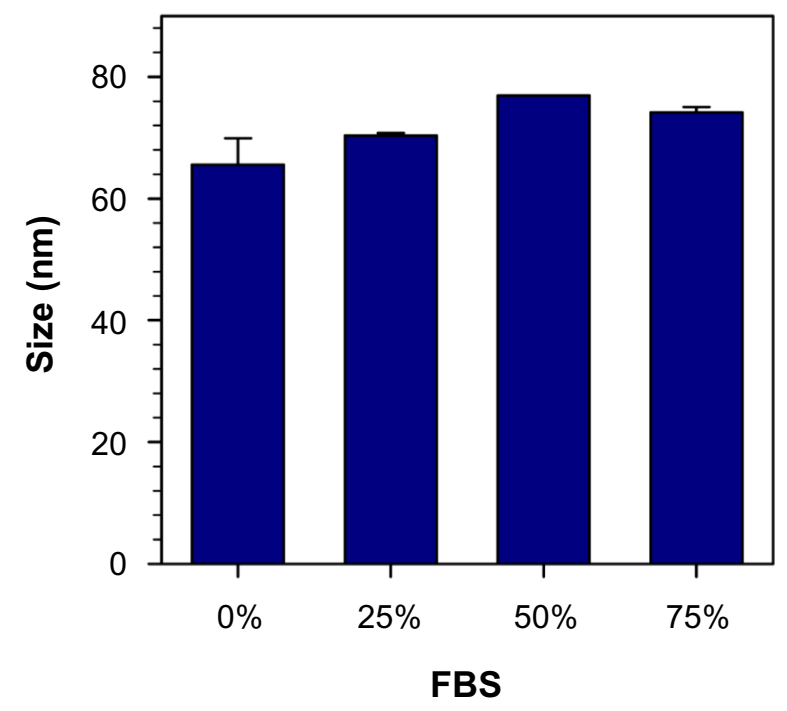

Figure 3 Average size of dextran-encrusted magnetic nanoclusters in various concentrations of $\mathrm{FBS} 0 \%, 25 \%, 50 \%$ and $75 \%$.

Abbreviation: FBS, fetal bovine serum. may have been due to the presence of van der Waals forces during the DMNC drying process. ${ }^{48,49}$ To confirm colloidal stability, DMNCs were redispersed in various concentrations of fetal bovine serum $(0 \%, 25 \%, 50 \%$, and $75 \%)$ and their average size was then analyzed by dynamic laser scattering. As shown in Figure 3, the sizes were very similar, confirming that the DMNCs had high stability independent of serum concentration.

Thermogravimetric analysis was performed to assess the quantity of the pyrenyl dextran layer. The quantity of magnetic nanoclusters was approximately $87 \%$ and the quantity of the organic fraction was found to be $13 \%$ (Figure 4A). $\mathrm{X}$-ray diffraction patterns were determined for the DMNCs, and their diffraction peaks closely resembled those of $\mathrm{Fe}_{3} \mathrm{O}_{4}$ (JCPDS 10-319), suggesting the existence of a mixed spinel structure and high crystallinity in the DMNCs, irrespective of the presence of pyrenyl dextran (Figure 4B).
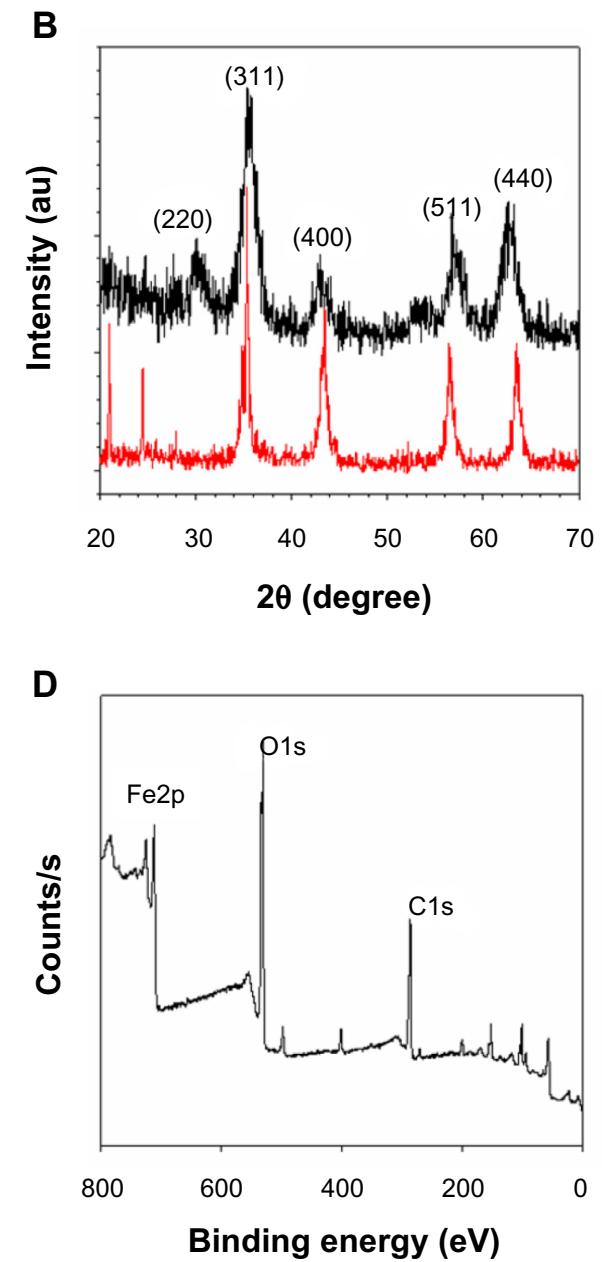

Figure 4 (A) Thermogravimetric analysis, (B) X-ray diffraction patterns of magnetic nanoclusters (black) and dextran-encrusted magnetic nanoclusters (red). (C) Magnetic hysteresis loops of dextran-encrusted magnetic nanoclusters at $273 \mathrm{~K}$, and (D) elemental composition of the surface using X-ray photoelectron spectroscopy of dextran-encrusted magnetic nanoclusters. 
A

\begin{tabular}{|c|c|c|c|c|c|}
\hline $\begin{array}{c}\text { Concentration } \\
(\mu \mathrm{M})\end{array}$ & 100 & 50 & 25 & 12.5 & 6.25 \\
\hline $\begin{array}{c}\text { T2-weighted MR } \\
\text { image }\end{array}$ & & & & & \\
\hline
\end{tabular}

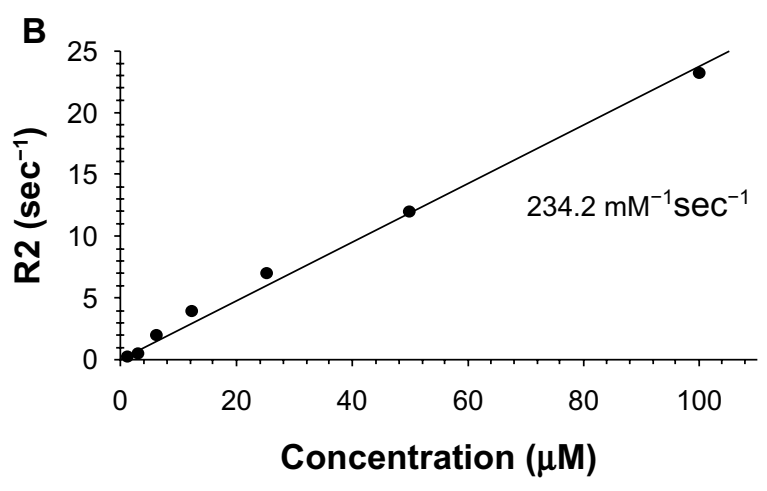

Figure 5 (A) T2-weighted MR images and (B) relaxivity (R2) graph of dextranencrusted magnetic nanoclusters at various concentrations. Abbreviation: MR, magnetic resonance.

\section{Magnetization and $\mathrm{T} 2$ relaxivity of DMNCs}

To evaluate the magnetic response of DMNCs to an external field, saturation of magnetization was measured at $298 \mathrm{~K}$. The superparamagnetic behavior of DMNCs was indicated by the absence of a hysteresis loop; their saturation magnetization was measured to be $60.1 \mathrm{emu} / \mathrm{g}_{\mathrm{Fe}}$ (Figure 4C). In addition, X-ray photoelectron spectroscopy demonstrated

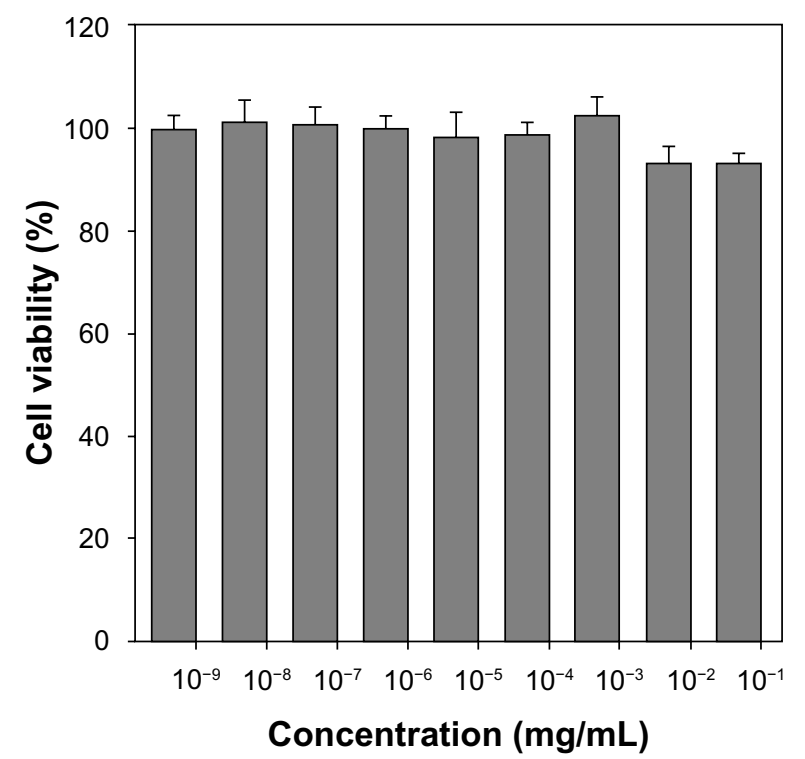

Figure 6 Viability of RAW264.7 cells treated with various concentrations of dextran-encrusted magnetic nanoclusters.
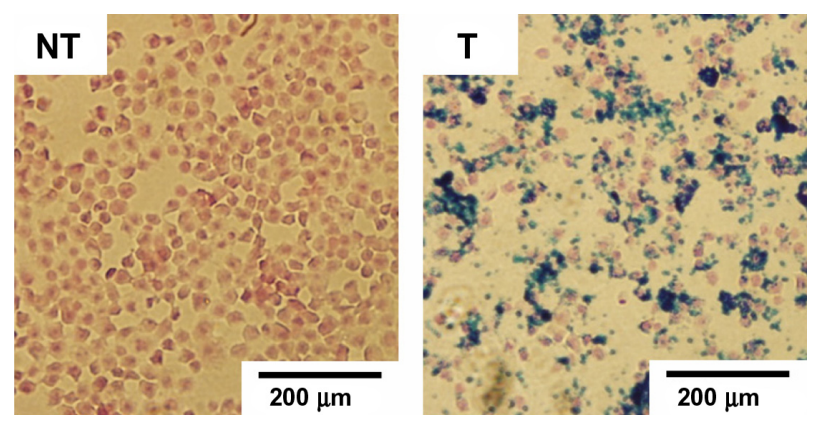

Figure 7 Prussian blue-stained microscopic images of RAW264.7 cells treated without and with dextran-encrusted magnetic nanoclusters.

Abbreviations: NT, nontreated; T, treated.

the presence of oxygen $(\mathrm{O})$ and carbon $(\mathrm{C})$ element spectra on the surface (Figure 4D). The element iron was barely detected (4.68\%), indicating successful encrusting of iron within the pyrene dextran. The potential of the DMNCs to act as an MR contrast agent was estimated by evaluating their MR signal intensity. Figure 5A shows the T2-weighted MR image along with the color map displaying a decrease in intensity as the DMNC concentration decreased. This behavior demonstrates that DMNCs can be used as effective T2 MR contrast agents. The $r^{2}$ value was calculated to be $234.2 \mathrm{mM}^{-1} \mathrm{sec}^{-1}$ (Figure 5B).

\section{Cytotoxicity assay and cellular uptake of DMNCs}

Cytotoxicity of the DMNCs was examined by MTT assay using a RAW264.7 macrophage cell lines. Cell viability was more than $80 \%$ on exposure to DMNCs, with no disruption of cell proliferation, even at a high concentration $\left(9.0 \mathrm{mg}_{\mathrm{Fe}} / \mathrm{mL}\right.$, Figure 6). Next, the macrophage cell line was treated with DMNCs to investigate cellular internalization. Prussian blue staining was used to visualize the iron content in the DMNC-treated cells. An acid solution of ferrocyanides in
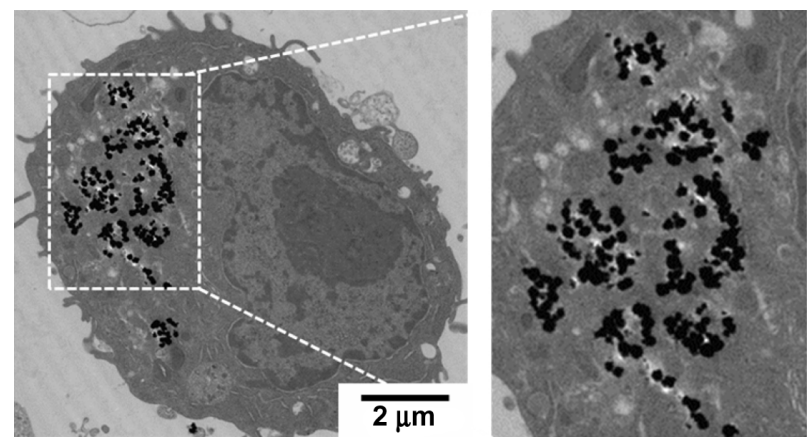

Figure 8 Transmission electron microscopic images of RAW264.7 cells treated with dextran-encrusted magnetic nanoclusters. 

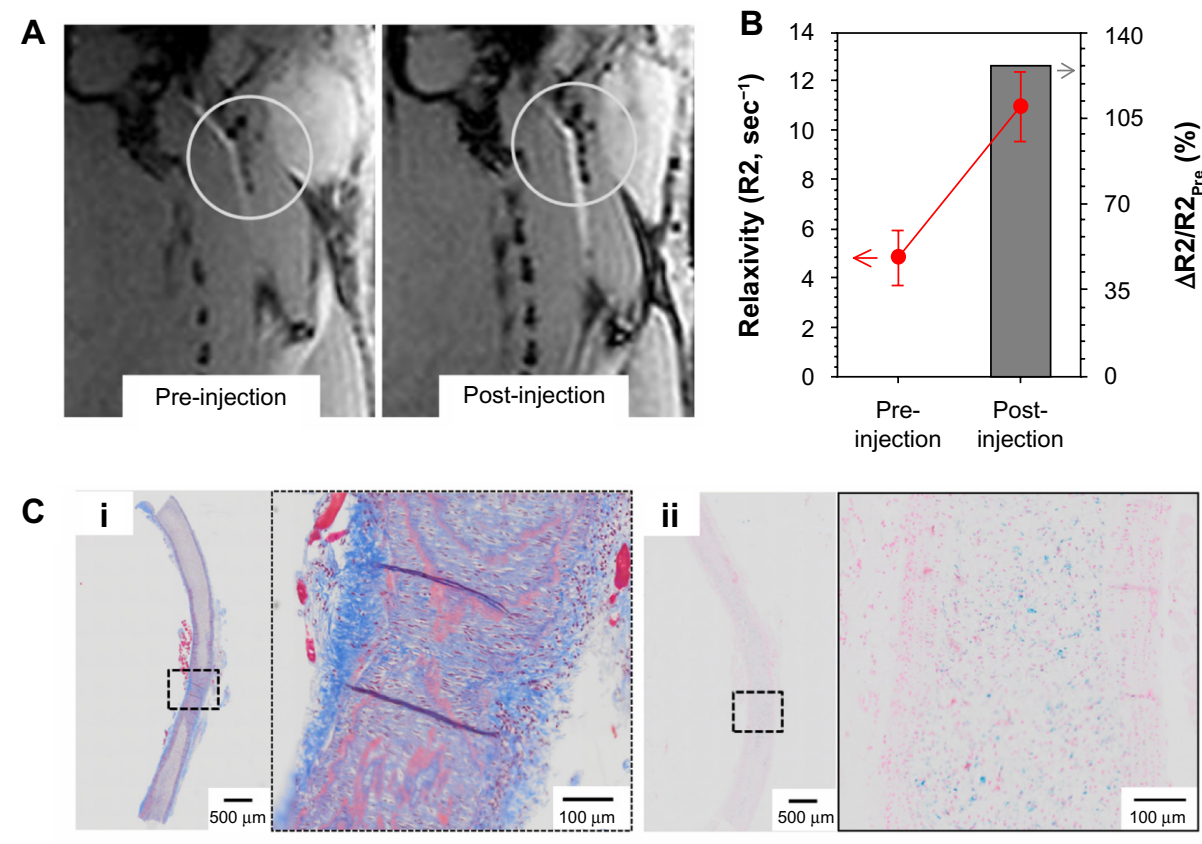

Figure 9 (A) In vivo magnetic resonance images before and after injection of dextran-encrusted magnetic nanoclusters, (B) relaxivity (R2, red circle) and $\Delta \mathrm{R} 2 / \mathrm{R} 2_{\text {pre }}(\%$, gray bar) graph (before: pre-injection and after: post-injection), and (C) histological staining images of the common carotid artery; (i) Mason's trichrome image and (ii) Prussian blue-stained image. The images on the right indicate the dashed quadrangle section of the entire image at high magnification.

Prussian blue stain combines with any ferric (III) ion present in the cell, leading to formation of a bright blue pigment. In Figure 7, prominent blue spots indicated significant binding of DMNCs to the cells. In comparison, blue spots were not observed in nontreated cells. A large number of black clusters were observed in the cytoplasm of the macrophage cells, indicating engulfment of DMNCs via a specific interaction which did not damage the cell nucleus (Figure 8).

\section{In vivo $M R$ imaging study}

We then performed in vivo MR imaging experiments to investigate the targeting capability of the DMNCs and their potential to enhance the MR image contrast. A rat balloon carotid injury model was used for this experiment. MR images were obtained before and up to 3 hours after intravenous injection of $4.8 \mathrm{mg}_{\mathrm{Fe}} / \mathrm{kg}$, as shown in Figure $9 \mathrm{~A} .{ }^{50} \mathrm{As}$ soon as the DMNCs were administered, the atherosclerotic lesion became noticeably darker, with a high MR signal, reaching the maximal $\Delta \mathrm{R} 2 / \mathrm{R} 2_{\text {Pre }}(\%)$ value of approximately $126.5 \%$ (Figure 9A), indicating that DMNCs were delivered successfully and accumulated in the lesion. After the MR study, the common carotid arteries were excised, and the presence of DMNCs on the interior wall of the common carotid artery was confirmed by histological staining (using Masson's trichrome and Prussian blue stains) to explore the correlation with in vivo MR images. Figure 9C(i) shows thrombus and its adhesion in the injured common carotid artery. The blue spots, which indicate the iron content in the DMNCs, were particularly observed in thrombus-induced lesions, as shown in Figure 9C(ii). These results indicate that DMNCs can provide MR signal enhancement in detection of atherosclerosis by accumulating in large quantities in macrophages because of the strong interaction between dextran and the scavenger receptor of the macrophage.

\section{Conclusion}

We have developed a one-pot solution phase synthetic method for water-soluble DMNCs that can be used to detect atherosclerosis by targeted MR imaging. The as-synthesized particles showed high colloidal stability and low cytotoxicity as well as high saturation magnetization at room temperature. Additionally, in vitro and in vivo MR studies demonstrated the suitability of DMNCs as effective contrast agents. Definitive diagnosis of atherosclerosis may require macrophage-based detection, and with further optimization, the results of this study may potentially have clinical application.

\section{Acknowledgments}

This study was supported by grants from the Bio and Medical Technology Development Program of the National Research Foundation funded by the Korean government (2012050077) and Ministry of Education Science and 
Technology (2012-2043991), and the KRCF Research Initiative Program.

\section{Disclosure}

The authors report no conflicts of interest in this work.

\section{References}

1. Jun YW, Huh YM, Choi JS, et al. Nanoscale size effect of magnetic nanocrystals and their utilisation for cancer diagnosis via magnetic resonance imaging. J Am Chem Soc. 2005;127(16):5732-5733.

2. Huh YM, Jun YW, Song HT, et al. In vivo magnetic resonance detection of cancer by using multifunctional magnetic nanocrystals. J Am Chem Soc. 2005;127(35):12387-12391.

3. Jun YW, Seo JW, Cheon J. Nanoscaling laws of magnetic nanoparticles and their applicabilities in biomedical sciences. Acc Chem Res. 2008;41(2):179-189.

4. Lee JH, Huh YM, Jun YW, et al. Artificially engineered magnetic nanoparticles for ultra-sensitive molecular imaging. Nat Med. 2007;13(1):95-99

5. Lu J, Ma S, Sun J, et al. Manganese ferrite nanoparticle micellar nanocomposites as MRI contrast agent for liver imaging. Biomaterials. 2009;30(15):2919-2928.

6. Weissleder R, Pittet MJ. Imaging in the era of molecular oncology. Nature. 2008;452(7187):580-589.

7. Sugimoto T, Matijević E. Formation of uniform spherical magnetite particles by crystallization from ferrous hydroxide gels. J Colloid Interface Sci. 1980;74(1):227-243.

8. Chen X, Sun X, Li Y. Self-assembling vanadium oxide nanotubes by organic molecular templates. Inorg Chem. 2002;41(17):4524-4530.

9. Harris LA, Goff JD, Carmichael AY, et al. Magnetite nanoparticle dispersions stabilized with triblock copolymers. Chem Mater. 2003;15(6):1367-1377.

10. Jolivet JP, Chaneac C, Tronc E. Iron oxide chemistry. From molecular clusters to extended solid networks. Chem Commun (Camb). 2004(5): 481-483.

11. Li F, Liu J, Evans DG, Duan X. Stoichiometric synthesis of pure $\mathrm{MFe} 2 \mathrm{O} 4(\mathrm{M}=\mathrm{Mg}, \mathrm{Co}$, and $\mathrm{Ni}$ ) spinel ferrites from tailored layered double hydroxide (hydrotalcite-like) precursors. Chem Mater. 2004;16(8):1597-1602.

12. Matijevic E. Monodispersed metal (hydrous) oxides - a fascinating field of colloid science. Acc Chem Res. 1981;14(1):22-29.

13. Sahoo Y, Cheon M, Wang S, Luo H, Furlani EP, Prasad PN. Field-directed self-assembly of magnetic nanoparticles. $J$ Phys Chem B. 2004;108(11):3380-3383.

14. Song Q, Zhang ZJ. Shape control and associated magnetic properties of spinel cobalt ferrite nanocrystals. J Am Chem Soc. 2004;126(19): 6164-6168.

15. Zhou ZH, Wang J, Liu X, Chan HSO. Synthesis of FeO nanoparticles from emulsions. J Mater Chem. 2001;11(6):1704-1709.

16. Jun YW, Lee JH, Cheon J. Chemical design of nanoparticle probes for high-performance magnetic resonance imaging. Angew Chem Int Ed Engl. 2008;47(28):5122-5135.

17. Sun S, Zeng H, Robinson DB, et al. Monodisperse MFe2O4 ( $\mathrm{M}=\mathrm{Fe}$, Co, Mn) nanoparticles. J Am Chem Soc. 2003;126(1):273-279.

18. Amstad E, Gillich T, Bilecka I, Textor M, Reimhult E. Ultrastable iron oxide nanoparticle colloidal suspensions using dispersants with catechol-derived anchor groups. Nano Lett. 2009;9(12): $4042-4048$

19. Ai H, Flask C, Weinberg B, et al. Magnetite-loaded polymeric micelles as ultrasensitive magnetic-resonance probes. Adv Mater. 2005;17(16): 1949-1952

20. Barrera C, Herrera AP, Rinaldi C. Colloidal dispersions of monodisperse magnetite nanoparticles modified with poly(ethylene glycol). J Colloid Interface Sci. 2009;329(1):107-113.
21. Basti H, Ben Tahar L, Smiri LS, et al. Catechol derivatives-coated $\mathrm{Fe} 3 \mathrm{O} 4$ and $\gamma$-Fe2O3 nanoparticles as potential MRI contrast agents. $J$ Colloid Interface Sci. 2010;341(2):248-254.

22. Fang C, Bhattarai N, Sun C, Zhang M. Functionalized nanoparticles with long-term stability in biological media. Small. 2009;5(14): 1637-1641.

23. Qin J, Laurent S, Jo YS, et al. A high-performance magnetic resonance imaging T2 contrast agent. Adv Mater. 2007;19(14):1874-1878.

24. Xie J, Xu C, Kohler N, Hou Y, Sun S. Controlled PEGylation of monodisperse $\mathrm{Fe} 3 \mathrm{O} 4$ nanoparticles for reduced non-specific uptake by macrophage cells. Adv Mater. 2007;19(20):3163-3166.

25. Yang HM, Lee HJ, Park CW, et al. Endosome-escapable magnetic poly(amino acid) nanoparticles for cancer diagnosis and therapy. Chem Commun (Camb). 2011;47(18):5322-5324.

26. Yang HM, Park CW, Lim S, Park SI, Chung BH, Kim JD. Cross-linked magnetic nanoparticles from poly(ethylene glycol) and dodecyl grafted poly(succinimide) as magnetic resonance probes. Chem Commun (Camb). 2011;47(46):12518-12520.

27. Cheng FY, Su CH, Yang YS, et al. Characterization of aqueous dispersions of $\mathrm{Fe} 3 \mathrm{O} 4$ nanoparticles and their biomedical applications. Biomaterials. 2005;26(7):729-738.

28. Thode K, Lück M, Schröder W, Blunk T, Müller RH, Kresse M. The influence of the sample preparation on plasma protein adsorption patterns on polysaccharide-stabilized iron oxide particles and $\mathrm{N}$-terminal microsequencing of unknown proteins. J Drug Target. 1998;5(6): 459-469.

29. Feldmann C, Jungk HO. Polyol-mediated preparation of nanoscale oxide particles. Angew Chem Int Ed Engl. 2001;40(2):359-362.

30. Wan J, Cai W, Meng X, Liu E. Monodisperse water-soluble magnetite nanoparticles prepared by polyol process for high-performance magnetic resonance imaging. Chem Commun (Camb). 2007;47:5004-5006.

31. Choi R, Yang J, Choi J, et al. Thiolated dextran-coated gold nanorods for photothermal ablation of inflammatory macrophages. Langmuir. 2010;26(22):17520-17527.

32. Lim EK, Jang E, Kim B, et al. Dextran-coated magnetic nanoclusters as highly sensitive contrast agents for magnetic resonance imaging of inflammatory macrophages. J Mater Chem. 2011;21(33): $12473-12478$

33. Lim EK, Jang E, Kim J, et al. Self-fabricated dextran-coated gold nanoparticles using pyrenyl dextran as a reducible stabilizer and their application as CT imaging agents for atherosclerosis. J Mater Chem. 2012;22(34):17518-17524.

34. McCarthy JR, Jaffer FA, Weissleder R. A macrophage-targeted theranostic nanoparticle for biomedical applications. Small. 2006;2(8-9): 983-987.

35. Peiser L, Gordon S. The function of scavenger receptorsexpressed by macrophages and their rolein the regulation of inflammation. Microbes Infect. 2001;3(2):149-159.

36. Xie J, Peng S, Brower N, Pourmand N, Wang SX, Sun S. One-pot synthesis of monodisperse iron oxide nanoparticles for potential biomedical applications. Pure Appl Chem. 2006;78(5):1003-1014.

37. Jaffer FA, Libby P, Weissleder R. Molecular and cellular imaging of atherosclerosis emerging applications. J Am Coll Cardiol. 2006;47(7): 1328-1338.

38. Xuan S, Wang F, Wang YXJ, Yu JC, Leung KCF. Facile synthesis of size-controllable monodispersed ferrite nanospheres. J Mater Chem. 2010;20(24):5086-5094.

39. Mosmann T. Rapid colorimetric assay for cellular growth and survival: application to proliferation and cytotoxicity assays. J Immunol Methods. 1983;65(1-2):55-63.

40. Furie B, Furie BC. Mechanisms of thrombus formation. NEngl J Med. 2008;359(9):938-949

41. Tulis D. Rat carotid artery balloon injury model. In: Sreejayan N, Ren J, editors. Vascular Biology Protocols. New York, NY, USA: Humana Press; 2007.

42. Dees C, Akhmetshina A, Zerr P, et al. Platelet-derived serotonin links vascular disease and tissue fibrosis. J Exp Med. 2011;208(5):961-972. 
43. Carson FL, Hladik C. Histotechnology: A Self-Instructional Text. Chicago, IL, USA: American Society of Clinical Pathologists; 1990.

44. Yin D, Wang Z, Gao Q, et al. Determination of the fate and contribution of ex vivo expanded human bone marrow stem and progenitor cells for bone formation by 2.3ColGFP. Mol Ther. 2009;17(11):1967-1978.

45. Deng H, Li X, Peng Q, Wang X, Chen J, Li Y. Monodisperse magnetic single-crystal ferrite microspheres. Angew Chem Int Ed Engl. 2005;44(18):2782-2785.

46. Ge J, Hu Y, Biasini M, Beyermann WP, Yin Y. Superparamagnetic magnetite colloidal nanocrystal clusters. Angew Chem Int Ed Engl. 2007;46(23):4342-4345.
47. Libert S, Gorshkov V, Goia D, Matijević E, Privman V. Model of controlled synthesis of uniform colloid particles: cadmium sulfide. Langmuir. 2003;19(26):10679-10683.

48. Çatiker E, Güner A. Unperturbed dimensions and the theta temperature of dextran in ethylene glycol solutions. Eur Polym J. 2000;36(10): 2143-2146.

49. Dou H, Tang M, Sun K. A facile one-pot synthesis to dextran-based nanoparticles with carboxy functional groups. Macromol Chem Phys. 2005;206(21):2177-2181.

50. Briley-Saebo KC, Mani V, Hyafil F, Cornily JC, Fayad ZA. Fractionated Feridex and positive contrast: in vivo MR imaging of atherosclerosis. Magn Reson Med. 2008;59(4):721-730. 


\section{Supplementary material}

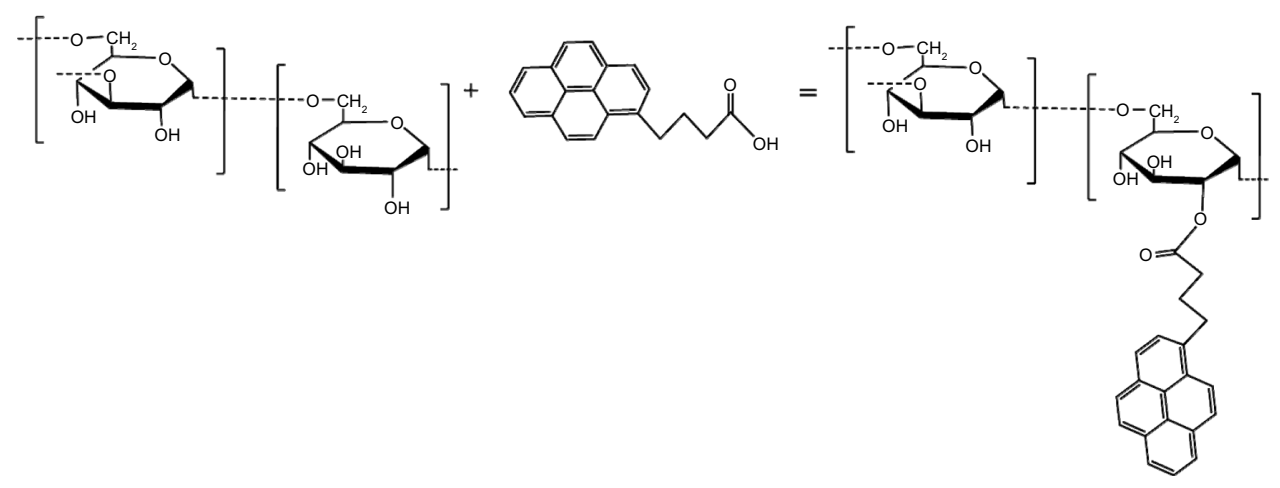

Figure SI Schematic illustration of synthesis of pyrenyl dextran.

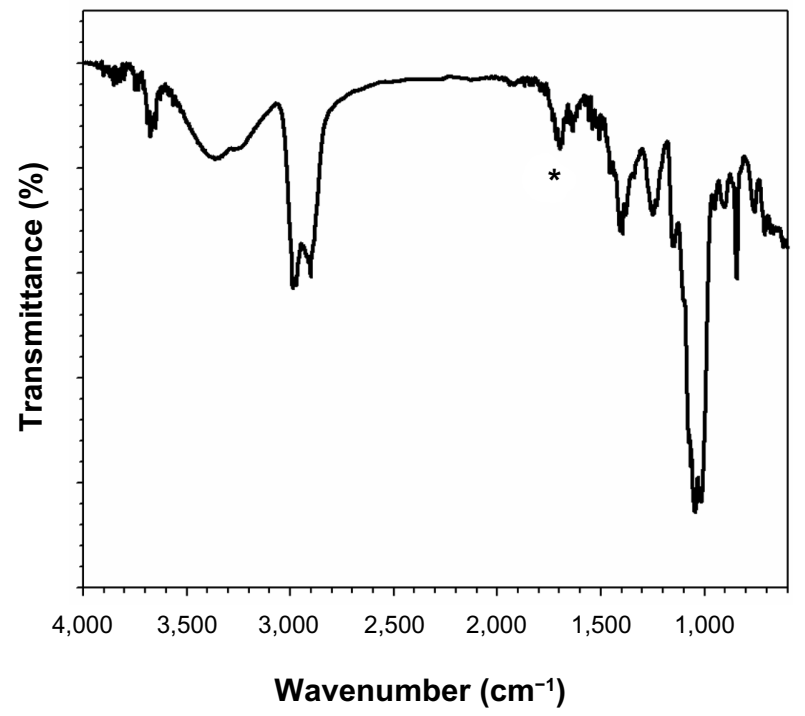

Figure S2 Fourier-transform infrared spectrum for pyrenyl dextran. Note: *Newly formed ester bond at $1,727 \mathrm{~cm}^{-1}$.

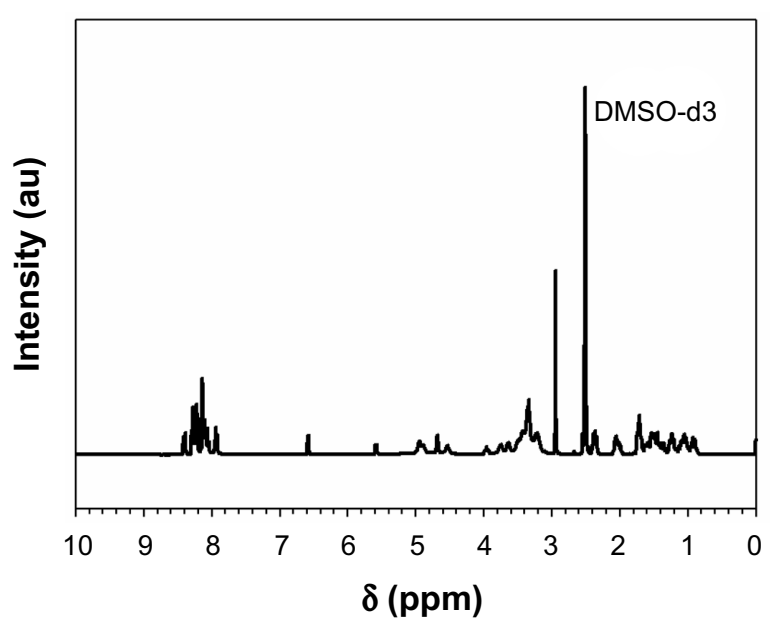

Figure S3 'H-nuclear magnetic resonance spectrum for pyrenyl dextran. Abbreviation: DMSO, dimethyl sulfoxide.

\section{Publish your work in this journal}

The International Journal of Nanomedicine is an international, peerreviewed journal focusing on the application of nanotechnology in diagnostics, therapeutics, and drug delivery systems throughout the biomedical field. This journal is indexed on PubMed Central, MedLine, CAS, SciSearch $\AA$, Current Contents $\AA /$ Clinical Medicine,
Journal Citation Reports/Science Edition, EMBase, Scopus and the Elsevier Bibliographic databases. The manuscript management system is completely online and includes a very quick and fair peer-review system, which is all easy to use. Visit http://www.dovepress.com/ testimonials.php to read real quotes from published authors. 